\title{
Compréhension orale et compréhension écrite: comment solidifier leurs liens et conserver leurs spécificités?
}

\section{Nancy Allen}

Les liens entre compréhension orale et compréhension écrite sont indéniables, même si des caractéristiques distinctes leur sont conférées. Dans cet article, nous proposons de décrire de manière qualitative des stratégies métacognitives verbalisées par six élèves du troisième cycle du primaire québécois (11-12 ans) dans un projet d'écoute. Dans ce dernier, ils ont été amenés à utiliser une grille d'écoute pour rendre compte de leur compréhension orale. Leurs verbalisations nous apprennent qu'ils utilisent essentiellement une stratégie de prise de notes pour retenir des informations. Ces données nous aident à penser à l'utilisation de cette stratégie dans une perspective transdisciplinaire d'enseignement.

\section{Introduction}

$\mathrm{Au}$ Québec, pour enseigner la compréhension orale, les enseignants peuvent s'appuyer sur la notion de projet d'écoute (Lafontaine, 2007) qui se divise en trois parties: la préécoute dans laquelle une intention d'écoute pédagogique est formulée pour orienter la prise de notes en vue de la réalisation d'un projet final; l'écoute globale, qui consiste à prendre connaissance du document audio ou audiovisuel sans prendre de note et l'écoute analytique lors de laquelle l'écoute est orientée en fonction de l'intention d'écoute puis; enfin, l'après-écoute qui consiste à réaliser un projet dans lequel les informations entendues sont réinvesties (une production écrite, un exposé oral, à titre d'exemple). Dans ces derniers, les élèves reçoivent généralement une grille d'écoute ${ }^{1}$ dont le découpage permet de noter les informations jugées essentielles pour répondre à l'intention d'écoute. On reconnait ainsi que pour retenir, comprendre et traiter les informations orales, les élèves ont besoin d'un support écrit pour les colliger et éviter de se retrouver en situation de surcharge cognitive.

Dans les classes du primaire québécoises, bien que la notion de genre soit présente dans les documents ministériels, cette dernière n'apparait pas dans le matériel prescrit pour l'enseignement de l'oral. Avec l'ouvrage Caractéristiques 
de 50 genres pour développer les compétences langagières en français, Chartrand, Émery-Bruneau et Sénéchal (2015) posent à peine les balises pour comprendre et orienter l'enseignement de l'oral, mais au secondaire. Ainsi, l'on constate que l'enseignement de la production et de la compréhension orales est peu présent en salle de classe. C'est en partie parce que la pédagogie, les stratégies et les techniques utilisées dans l'enseignement de l'oral sont empruntées à celles de l'écrit et que les enseignants peinent à décloisonner ces deux volets de la didactique (Allen, 2017a; Sénéchal \& Chartrand, 2011). Cela explique certaines des raisons pour lesquelles l'enseignement des objets de la compréhension orale repose sur ceux de la compréhension écrite, mieux connue et documentée, et la difficulté liée au manque de ressources pour lier les caractéristiques communes aux genres présents à l'oral et à l'écrit s'y ajoute. Les ramifications entre ces deux aspects de la compréhension sont possibles, si leurs caractéristiques propres ne sont pas occultées.

\section{La notion de genre à l'oral et à l'écrit}

La notion de genre à l'oral se définit chez Bronckart (1996, p. 74) comme «toute unité de production verbale véhiculant un message linguistiquement organisé et tendant à produire sur son destinataire un effet de cohérence». Dolz et Schneuwly (1998) diront pour leur part que «c'est au travers des genres que les pratiques langagières s'incarnent dans les activités des apprenants» (p. 29). Ainsi, il n'est pas tout à fait faux de dire que les codes de la compréhension orale et de la compréhension écrite sont apparentés (Garcia-Debanc \& Plane, 2004; Nonnon, 2002; Parpette, 2008) puisque les deux s'inscrivent dans un genre dont les contextes d'énonciations possèdent des caractéristiques communes. La syntaxe des propos est généralement respectée, le lexique l'est aussi, mais ce sont les conditions énonciatives de mise en bouche, c'est-à-dire les éléments prosodiques et parfois ceux aussi subjectifs, notamment la spontanéité, qui varient. De plus, la contextualisation des propos est parfois absente à l'oral, car il est admis que les interlocuteurs ont des référents communs; certaines reformulations de même que les éléments non verbaux sont quelques exemples qui montrent pourtant des différences entre les deux codes)..

L'absence de symétrie complète entre la compréhension orale et écrite a été démontrée (Garcia-Debanc \& Plane, 2004; Parpette, 2008) conférant du même coup à la compréhension orale ses objets d'enseignement singuliers, même s'ils s'apparentent à ceux de l'écrit. La compréhension orale, tout comme la compréhension écrite, sont des processus actifs au cours desquels le sens d'un message se construit (Parpette, 2008; Rost, 2002) dans le temps, selon divers facteurs. Comme cela est mis en place en situation d'écriture où un genre textuel est respecté, des stratégies métacognitives doivent être enseignées en contexte de compréhension orale afin que les élèves soient amenés à comprendre les 
caractéristiques communes aux deux types d'énonciations qui relèvent d'un même genre (Chiss, 2002; Cornaire \& Germain, 1998; Dolz \& Schneuwly, 1998).

\section{Les stratégies d'enseignement}

Plusieurs des stratégies de compréhension de documents écrits (donc en lecture) sont prescrites dans l'enseignement de la compréhension orale (par exemple, dans le document ministériel québécois Progression des apprentissages au primaire désormais [PDA] en lecture, ministère de l'Éducation, du Loisir et du Sport désormais [MELS], 2009) on indique "Comparer son appréciation avec celle d'autres personnes» ce qui peut trouver écho dans la stratégie en oral «Soutenir ou valoriser les propos d'autrui» (MELS, 2011). Toutefois, la compréhension orale comporte des niveaux de compréhension: l'élève doit interagir avec le document entendu, qui est pour sa part éphémère. Par la suite, il doit interpréter les éléments verbaux et non verbaux. Ces différences majeures convoquent des stratégies métacognitives de régulation et de gestion de la compréhension qui interviennent sur divers plans, notamment en raison de la fugacité de l'oral, ce que ne convoque pas tout à fait la compréhension écrite, en raison de son caractère statique. Ainsi, il s'avère nécessaire de faire des liens entre les stratégies métacognitives dans toutes les disciplines puisque la compréhension orale et la compréhension écrite y sont convoquées, ce que permet une entrée par les genres (Chartrand, ÉmeryBruneau \& Sénéchal, 2015). On assure ainsi le transfert des connaissances dans des contextes diversifiés, régi par des caractéristiques communes, ce qui, pour les élèves, augmente leurs chances de réinvestir et de mobiliser des stratégies métacognitives (Allen, 2017a; Vandergrift \& Goh, 2012).

\section{Bénéfices liés à l'enseignement de stratégies propres aux genres en compréhension orale et écrite}

Trois stratégies métacognitives sont à enseigner explicitement aux élèves afin qu'ils développent les compétences nécessaires à la réussite d'une tâche de compréhension écrite (Efklides, 2008; Fayol \& Gombert, 1992). Ces stratégies servent également pour une tâche de compréhension orale. D'abord, des stratégies d'orientation, qui permettent à l'élève de saisir l'enjeu et les consignes de la tâche, et des stratégies de planification qui demandent de diviser la tâche (processus de bas niveau) de manière à définir les procédures à suivre pour la réussir (processus de haut niveau). L'élève doit ensuite réguler ses processus cognitifs et les ajuster au besoin au moyen de stratégies de vérification qui consistent à revoir la planification initiale de l'exécution de la tâche, selon l'intention donnée préalablement. 
L'utilisation de ces trois stratégies demande un entrainement assidu, car «savoir comment faire quelque chose ne signifie pas nécessairement que ces activités [sont] conscientes» (Brown \& Campione, 1995, p. 68). En effet, l'utilisation de stratégies peut être variable d'un élève à l'autre et même mise en place inconsciemment tel un automatisme (Beacco \& Porquier, 2007; Romainville, 2000). À la suite de ces étapes, l'élève peut évaluer son résultat à l'égard de la tâche demandée et convoquer des stratégies métacognitives telles que l'autorégulation pour identifier les forces et les défis pour réussir la tâche. Par exemple, dans la PDA en lecture, pour la compétence Lire des textes variés, l'élève doit «Évaluer sa démarche de lecture en vue de l'améliorer: se questionner sur l'efficacité des stratégies utilisées» (MELS, 2011)2. Cette stratégie s'apparente à "Réfléchir à la qualité de son écoute et de ses interventions» qui est présente sous la compétence Communiquer oralement. En effet, lors de la réalisation d'un projet d'écoute, l'élève doit pouvoir réfléchir au sujet de sa prise de notes et des stratégies métacognitives mises en place pour structurer les propos entendus, afin de répondre à l'intention d'écoute qui lui est donnée. À la lumière des prescriptions ministérielles, il semble impératif d'enseigner une stratégie de prise de notes succinctes en situation de compréhension orale, afin que les élèves retiennent et discriminent les informations essentielles (Bétrix Köhler, Nidegger, Revaz, Riesen \& Wirthner, 1999; de Pietro \& Whirthner, 1996; Letarte \& Lafond, 2001; Nolin, 2013; Nonnon, 2002). Cela prévaut d'ailleurs pour toutes les disciplines puisque la compréhension n'est pas exclusive à l'apprentissage de la langue.

\section{Quelques liens qui unissent les stratégies métacognitives de compréhensions orale et écrites}

Diverses stratégies métacognitives de compréhension orale et de compréhension écrite sont apparentées dans les documents ministériels québécois. La stratégie métacognitive de compréhension orale "Clarifier ses propos ou ses réactions" (MELS, 2011) peut trouver écho dans la stratégie métacognitive en lecture "Considérer les réactions d'une autre personne (ex.: commentaire, critique)» (MELS, 2009), puisque considérer ces dernières nécessite un ajustement de la part du destinataire. "Revenir au sujet lorsqu'on s'en éloigne» (MELS, 2011) prend forme dans la stratégie métacognitive en lecture «Se rappeler les contraintes du contexte (ex.: temps alloué, lieu, mode de réponse)» (MELS, 2009) puisque cette dernière implique aussi d'être conscient de la situation actuelle et de ses contraintes formelles, afin de les respecter. "Ajuster ses paroles en cas d'incompréhension (autorégulation) en reformulant, en paraphrasant ou en expliquant» (MELS, 2011, p.?) équivaut pour sa part à la stratégie métacognitive en lecture «Ajuster sa compréhension à la suite d'échanges» (MELS, 2009, p.?), car elle concerne l'idée de rétablir le sens des informations lues ou entendues. De même, 
«Prendre une posture d'écoute: explorer des moyens pour organiser et retenir l'information" (MELS, 2011) et la stratégie métacognitive en lecture "Garder des traces de ses choix pour suivre sa propre évolution" (MELS, 2009) explorent toutes deux l'idée de noter pour conserver des traces. Enfin, «Relever des éléments moins réussis de sa communication (métacommunication)» (MELS, 2011) correspond, pour sa part, aux stratégies métacognitives en lecture "Constater sa progression en tant que lecteur» et «Se questionner sur l'efficacité des stratégies utilisées» (MELS, 2009) puisque ces stratégies ont en commun d'amener l'élève à réfléchir à ses apprentissages.

On comprend alors que plusieurs stratégies métacognitives de compréhension orale trouvent leur écho dans les stratégies métacognitives de compréhension écrite (en lecture), qu'elles soient issues des recherches (Efklides, 2008; Fayol \& Gombert, 1992) ou des documents ministériels (MELS, 2011). Néanmoins, on sait peu ou pas comment s'opérationnalise l'utilisation de telles stratégies par les élèves en contextes de projets d'écoute et c'est ce que cette recherche souhaite décrire afin de comprendre les liens entre l'enseignement de la compréhension orale et de la compréhension écrite.

\section{Objectif de cette recherche}

Afin de contribuer à la mise en lumière des liens possibles entre les stratégies de compréhension orale et celles de compréhension écrite, cette recherche qualitative à visée compréhensive et exploratoire (Van der Maren, 2010) a décrit les stratégies verbalisées par des élèves du troisième cycle du primaire québécois dans des projets d'écoute.

\section{Méthodologie}

\section{Échantillon}

Six élèves d'une classe du troisième cycle du primaire (11-12 ans) de la région de l'Outaouais, au Québec, ont été recommandés par leur enseignante et sélectionnés en raison de leur volubilité et de leurs caractéristiques personnelles hétérogènes. On espérait ainsi que ces derniers explicitent les stratégies qu'ils connaissent ou mobilisent pour réaliser un projet d'écoute et répondre à l'intention d'écoute qui leur était formulée.

\section{Instrumentation}

Trois projets d'écoute ont été élaborés par la chercheuse. Leur contenu a été révisé par une didacticienne universitaire puis validé en classe par une enseignante. Ils ont ensuite été soumis individuellement aux élèves participant à cette recherche. Chacun des projets d'écoute a porté sur un genre informatif ${ }^{3}$, genre 
que les élèves du troisième cycle du primaire doivent maitriser et, pour chacun d'eux, les élèves ont reçu une grille d'écoute dans laquelle ils ont noté des informations qu'ils jugeaient importantes pour réussir l'intention d'écoute qui leur a été donnéé . Les élèves avaient déjà travaillé à l'aide de grilles d'écoute en classe et étaient familiers avec leur format. À la suite du projet d'écoute5, les élèves ont réalisé une tâche de verbalisation à haute voix sans rappel ${ }^{6}$ (Vermersch, 2011) qui servait à connaitre les stratégies utilisées pour noter les informations, les retenir et les organiser afin de répondre à l'intention d'écoute.

\section{Analyse}

Les verbalisations à haute voix des élèves à la suite des projets d'écoute ont été filmées puis leurs propos ont été retranscrits. Ces derniers ont été codifiés manuellement et analysés avec l'analyse de contenu avec catégories fixes et émergentes (Paillé \& Muchielli, 2012). Les catégories fixes ont été créées à l'aide des stratégies présentes dans les documents ministériels québécois en oral qui servaient de répertoire de base pour construire les connaissances des élèves. Les catégories émergentes, pour leur part, contiennent les propos des élèves qui correspondent à l'utilisation de stratégies personnelles, c'est-à-dire des stratégies qui ne sont pas listées dans les documents ministériels. Ces stratégies permettent toutefois de comprendre les moyens mis en place par les élèves pour réaliser un projet d'écoute.

\section{Résultats}

Le premier projet d'écoute portait sur un sujet connu de chacun des élèves ${ }^{7}$ et ressemble aux tâches d'oral vécues en salle de classe pour lesquelles peu d'enseignement a généralement lieu - car, il faut le rappeler, l'oral est surtout un médium d'enseignement dans les classes du primaire (écouter un document et s'exprimer sur ce dernier par la suite, à l'oral ou à l'écrit) (Allen, 2017b; Beacco \& Porquier, 2007; Lhote, 1995). Afin d'obtenir un portrait naturel des connaissances des élèves au sujet des stratégies, ces derniers n'ont pas reçu d'enseignement avant de commencer le projet d'écoute. On s'attendait à ce qu'ils utilisent leur grille d'écoute sans pour autant comprendre comment sélectionner ce qu'ils pouvaient y noter.

\section{La prise de note: entre cognitif et métacognitif}

Lors de la réalisation du premier projet d'écoute, les élèves devaient s'exprimer et raconter ce qu'ils avaient entendu, mais ils pouvaient difficilement expliquer comment ils s'y étaient pris pour retenir les informations, autrement qu'en recourant à la stratégie "Prendre des notes». En effet, les élèves ont pris des notes, car l'intention d'écoute qui leur a été donnée (voir note 4) était: «Informer la chercheuse du sujet de la vidéo en s'appuyant seulement sur les informations du document audiovisuel». Fait à noter, les élèves utilisent tous cette stratégie, sans pour autant tous la verbaliser, comme si, en conséquence des réalités d'ensei- 
gnement de toutes les disciplines dans les salles de classe, les élèves prennent des notes, sans trop y réfléchir. Pour cette raison, cette stratégie est parfois cognitive (Chinn \& Chinn, 2009; Flavell, 1985; Hartman, 2001), c'est-à-dire qu'elle sert à noter toutes les informations sans juger de leur pertinence (ce fut notamment le cas pour la prise de notes de Constance qui notait même les déterminants). La stratégie «Prendre des notes» devient toutefois métacognitive, lorsque les élèves utilisent deux stratégies présentes dans les documents ministériels soit la stratégie métacognitive «Dégager des liens entre les propos échangés: enchainer sur les propos des autres» et la stratégie métacognitive «Prendre une posture d'écoute: explorer des moyens pour organiser et retenir l'information» (MELS, 2011) qui permettent de réfléchir aux informations entendues dans le projet d'écoute. En effet, la combinaison de ces stratégies permet aux élèves de sélectionner et de faire des liens entre les informations notées. Une stratégie similaire, selon l'intention d'utilisation qu'elle revêt, passe donc de la rétention de connaissances à l'organisation et à l'interprétation de ces dernières et ce glissement rappelle les différences qui subsistent entre la compréhension orale et celle de l'écrit. En situation de compréhension orale, ce sont les notes prises par les élèves qui font que ces dernières ont du sens et qui fixent les informations essentielles pour répondre à l'intention d'écoute.

\section{La prise de notes ne servirait qu'à retenir les informations entendues...}

Charlotte, Marguerite et Constance ont affirmé prendre des notes pour ne rien oublier, en espérant avoir tout noté pour répondre aux questions qui leur seraient ensuite posées. Elles ont répété reproduire leur comportement de prise de notes de la salle de classe. Cela correspond tant aux stratégies qu'elles ont verbalisées, à leur manière de noter les informations (phrases complètes, pourcentages, etc.) et d'organiser ces dernières. Comme les élèves se font régulièrement répéter en classe de prendre des notes pour ne rien oublier, il est compréhensible qu'ils aient peu conscience des processus liés à leur écoute (Lazure, 1992; Nonnon, 2004) et qu'ils aient recours essentiellement à cette stratégie, sans se questionner davantage sur les effets qu'elle peut avoir sur leur compréhension orale. En effet, dans les notes des élèves participant à cette recherche, aucune ne concerne leurs impressions, leurs sentiments et les effets mêmes de l'écoute. Toutes les notes prises sont factuelles. Cela permet de "comprendre» les informations, mais pas de les interpréter et de se les approprier (Berner \& Thouard, 2008; Vanderdope, 1992), ce qui ne représente pas un lien clair avec la compréhension écrite ou orale pour laquelle on répète aux élèves que les textes font "vivre des émotions». En effet, en situation de compréhension orale du quotidien, on est souvent prompt à relever des éléments du non verbal: un sourire, un sourcillement, des bras croisés. Ces éléments participent de la compréhension orale et ajoutent aux informations strictement verbales. Il en va de même pour les procédés prosodiques. Cependant, ces éléments n'ont pas été relevés par les élèves. Lors de l'entretien de sélection des élèves, certains ont pourtant nommé l'importance de 
ceux-ci pour mieux saisir les propos et leur portée. Or, à la suite du visionnement de la vidéo de Constance qui réalise le projet d'écoute, on remarque qu'elle lève à de très rares occasions les yeux vers la vidéo, se contentant d'écouter les propos...

L'interprétation et le jugement en situation de compréhension orale ne sont pas présents dans les indicateurs des documents ministériels pour l'oral, mais ils le sont pour la compréhension de textes écrits. On indique dans la PDA (MELS, 2009) sous la compétence Apprécier des œuvres littéraires que «Le processus d'appréciation s'appuie sur le processus de lecture tripartite (compréhension - interprétation - réaction), mais il requiert également une certaine distanciation afin de permettre l'expression du jugement. ${ }^{\star}$ Or, en contexte de compréhension orale, les élèves sont peu sollicités pour s'investir personnellement dans la tâche, alors que cela leur permettrait d'être plus engagés (Guthrie, Wigfield, Metsala \& Cox, 1999) et de mieux comprendre les liens entre l'utilisation de stratégies métacognitives d'une discipline à l'autre pour ainsi s'approprier les propos. Bien que le genre informatif ne nécessite pas des élèves qu'ils interprètent les propos au sens de Vanderdope (1992), par exemple, ces derniers doivent néanmoins relever les éléments non verbaux et prosodiques pour se construire une compréhension cohérente de la situation d'écoute.

Par leurs prises de notes, Charlotte, Marguerite et Constance semblent conscientes du caractère éphémère de l'oral et des possibles oublis qui lui sont attribuables (Nonnon, 2004; Parpette, 2008). Parce qu'elles réfléchissent à la qualité de leur écoute et qu'elles regroupent les informations entendues et retranscrites, elles gèrent leur compréhension. Toutefois, Constance note plusieurs informations littérales sur sa grille d'écoute, mais mentionne pourtant sélectionner les informations importantes» dans ce que j'entends et je note juste ça». Cette élève ne semble pas réfléchir aux fonctions de la prise de notes et répète probablement ce qu'on lui a déjà dit au sujet d'une prise de notes efficace, car ses propos ne reflètent pas les informations qu'elle a transcrites. Par ailleurs, tous les élèves, sauf Claire et parfois Octave, s'attardent à noter plusieurs informations plutôt qu'à sélectionner les plus pertinentes. Constance mentionne qu'elle souhaiterait un accompagnement plus explicite afin de savoir précisément quoi noter: "J'aurai aimé que tu me montres quoi dire et quoi faire. Moi, j’ai besoin de plus d'exemples que les autres à cause de mon TDA9". Elle ne semble pas consciente que les stratégies, de tout ordre, sont adaptatives en fonction de la tâche donnée et que leur utilisation peut être personnelle (Beacco \& Porquier, 2007). À cet effet, il appert que des élèves qui ont un sentiment d'auto-efficacité faible ont plus de difficultés à mettre en œuvre des conduites autorégulatoires appropriées (Bouffard, 1998), comme cela a semblé être le cas pour cette élève.

La difficulté d'exprimer les stratégies s'est également produite lors de la prise de notes de Marguerite qui comportent, en abondance, des informations importantes pour l'intention donnée, afin de ne rien oublier. Elle note en effet sans respecter l'intention d'écoute. À la suite de sa prise de notes, elle devait choisir les informations pertinentes à présenter et les interpréter, en les remettant en 
contexte, c'est-à-dire en répondant à l'intention d'écoute. Puisqu'elle avait noté trop d'informations, elle n'y est pas arrivée. Les autres élèves, sauf Octave, ont aussi noté abondamment les informations entendues, mais l'ont fait de manière plus systématique, ce qui a facilité le recours à leurs notes lors de la verbalisation à haute voix.

\section{Discussion}

En contexte scolaire, comprendre oralement signifie accéder au sens (Legendre, 2005; Wallace \& Louden, 2003) et, pour cela, les propos des élèves sur le contenu de la vidéo présentée lors du projet d'écoute sont justes; chacun, à leur manière, ils ont su en rappeler des éléments du contenu et ils ont tenté de répondre à l'intention d'écoute qui leur était formulée. Cependant, puisque coordonner l'écoute d'un document à une tâche à réaliser est cognitivement lourd pour les élèves, surtout s'il s'agit de réfléchir au-delà de la simple remémoration d'informations, il est compréhensible qu'ils éprouvent des difficultés dans la régulation métacognitive de la tâche (Graham, Santos \& Vanderplank, 2011), notamment en ce qui concerne la prise de notes. En effet, les élèves de cette recherche se sont peu approprié le document qui leur a été présenté. Ils se sont contentés de reprendre textuellement les informations entendues, plutôt que de se les approprier en les reformulant, par exemple, ce qui témoignerait d'une compréhension efficace. Or, si ces élèves maitrisent les caractéristiques du genre informatif à l'écrit (lecture), ils doivent arriver à faire siennes les informations pour les retranscrire, les paraphraser, etc. En ce sens, il s'avère que les liens entre la compréhension orale et la compréhension écrite sont difficilement transférés entre les deux volets de la compréhension. Soit les élèves n'ont pas su noter efficacement, c'est-à-dire noter seulement les informations clés, en fonction de l'intention d'écoute, soit ils ont difficilement su s'approprier les informations écrites dans leur grille d'écoute et s'en détacher, comme le prescrivent les documents ministériels pour le troisième cycle du primaire.

La mobilisation d'une stratégie métacognitive, comme l'anticipation, est absente des documents ministériels sous la compétence Communiquer oralement. Elle est toutefois listée dans les composantes de la lecture et doit être instaurée dès le début du primaire («Survoler le texte pour anticiper le contenu», MELS, 2006, 2011). Marguerite, qui verbalise cette stratégie en ce qui concerne sa prise de notes, est donc capable de réfléchir à la tâche qui lui est demandée et de gérer les étapes pour accomplir cette dernière, mais elle demeure à l'étape de la planification de la prise de notes et de la mémorisation des informations entendues. À ce propos, elle nous dit: «Je regarde les catégories de notes que je dois prendre sur ma feuille et ensuite je me questionne pour savoir quoi écrire, ce qui sera important et aussi je pense à ce que tu m'as dit, tsé, pourquoi j'écoute le vidéo». Son utilité réside dans la transférabilité qu'elle revêt pour d'autres disciplines et 
qui permet de diminuer les efforts cognitifs éprouvés par les élèves. Ainsi, un enseignement de la compréhension orale qui repose sur les genres, le genre informatif ici, trouve sa pertinence dans ses caractéristiques intrinsèques. Quoi noter? Comment le noter? Quelles informations verbales permettent de comprendre le propos? Qu'en est-il des éléments non verbaux et prosodiques pour cette compréhension? Lors d'une compréhension en lecture, ces questions sont posées aux élèves afin de mobiliser des stratégies de prédiction ou d'anticipation, lors d'une étape préalable de réactivation des connaissances. L'horizon d'attente (Lhote, 1995) des élèves doit aussi être développé pour la compréhension orale en insistant aussi sur les éléments non verbaux et prosodiques.

Par ailleurs, un enseignement qui s'appuie sur les genres permet aussi de cerner les raisons pour lesquelles une stratégie de prise de notes est nécessaire dans les autres disciplines. Les stratégies métacognitives verbalisées par les élèves ont aussi des ramifications dans d'autres disciplines scolaires et sont listées dans la PDA (MELS, 2009). Par exemple, dans le programme en Science et technologie 10 («Exploration: anticiper les résultats de sa démarche; faire appel à divers modes de raisonnement [ex.: induire, déduire, inférer, comparer, classifier»]). "Se faire des images mentales» est, quant à elle, présente dans le domaine des mathématiques ${ }^{11}$ (MELS, 2009) («Élaboration: puis-je me représenter la situation mentalement ou par écrit?»). "Gérer sa compréhension» est pour sa part présente dans la composante de la compétence Lire des textes variés12 (MELS, 2009) ("Ajuster sa compréhension à la suite d'échanges») et dans celle du domaine des mathématiques ${ }^{13}$ (MELS, 2009) ("Régulation: ai-je choisi une bonne stratégie et pris le temps nécessaire pour bien comprendre le problème» et "Ai-je une bonne démarche et puis-je l'expliquer?»). Pour pallier les difficultés verbalisées par les élèves au sujet de leur prise de parole sur les stratégies de compréhension orale, il importe qu'ils soient amenés à prendre conscience que ces dernières ne sont pas circonscrites à une seule situation scolaire (Beacco \& Porquier, 2007; Lafontaine, 2007).

Néanmoins, à travers les résultats issus des analyses, il semble qu'en dépit des documents ministériels (MELS, 2006, 2011) et des connaissances théoriques au sujet des stratégies de compréhension, les stratégies de compréhension orale demeurent reliées à un contexte voisin de la compréhension écrite et les élèves, surtout ceux présentant des difficultés d'apprentissage, prennent peu conscience des habiletés développées dans une situation pour les transférer dans une autre (Field, 2013; Graham, 2005), alors qu'elles sont pourtant adaptables (Beacco \& Porquier, 2007; Cain, Oakhill, Barnes \& Bryant, 2001). En outre, même si le Réseau canadien de recherche sur le langage et l'alphabétisation (2009) recommande aux enseignants d'aider les élèves à prendre conscience de leurs stratégies de pensée pour opérer un retour réflexif sur leurs processus d'apprentissage afin de mieux les maitriser, les verbalisations des élèves tendent à montrer que cela est limité puisqu'ils ont affirmé ne pas avoir reçu de guidage enseignant dans leur parcours scolaire. Il faut cependant nuancer cette affirmation en la 
considérant comme des propos rapportés. La nécessité d'instaurer des réflexions autour de la compréhension orale s'accroit donc pour les élèves qui réfléchissent peu à leurs processus métacognitifs ou qui éprouvent des difficultés cognitives (OCDE, 2014), car tous peuvent bénéficier d'un guidage afin de développer de nouvelles connaissances et, conséquemment, développer des liens entre les stratégies métacognitives de compréhension orale et celles de compréhension écrite. C'est d'ailleurs ce que soutient un enseignement par les genres.

Les élèves doivent être accompagnés pour reconnaitre et mobiliser des stratégies métacognitives, c'est-à-dire des stratégies leur permettant de s'interroger sur leurs processus mentaux lors de la réalisation d'une activité de compréhension orale, afin d'être conscientisés à leurs différents usages dans des contextes stimulants et variés, cela va de soi. Les documents ministériels offrent une place considérable aux objets d'enseignement de la communication orale, et la considèrent même comme une compétence transversale. Toutefois la faible présence d'indicateurs précis pour l'enseigner accentue les défis que vivent les enseignants qui consultent les documents ministériels et qui tentent des rapprochements parfois hasardeux avec la compréhension écrite, alors que leurs caractéristiques different... mais pas tant. En effet, une entrée par les genres permettrait même de faire des liens explicites entre les caractéristiques des deux compréhensions, ce qui favoriserait sûrement une mobilisation de stratégies métacognitives de la part des élèves.

\section{Conclusion}

Considérer les stratégies de compréhension orale dans une perspective essentiellement transversale répond aux Domaines généraux de formation du Programme de formation de l'école québécoise (MELS, 2006). L'importance accordée aux apprentissages en lecture et en écriture pour rehausser le niveau de littératie des élèves est indéniable (Conseil supérieur de la langue française, 2015). Il importe également de se rappeler que l'oral est un facteur de réussite - puisqu'il réunit les dimensions scolaire (réception et traitement des connaissances) et sociale (interactions avec les autres) dans les apprentissages. Il nécessite que l'on s'y penche plus avant et que l'on s'efforce de développer des compétences en compréhension orale chez les élèves, comme on s'y penche d'ailleurs dans les autres disciplines scolaires, mais surtout que les stratégies de compréhension orale soient enseignées en concordance avec celles des autres disciplines, comme une entrée par les genres le permettrait.

Cette recherche, dont les résultats sont centrés sur l'élève, permet de penser autrement la compréhension orale. En effet, l'incidence de cette dernière n'est pas seulement liée aux apprentissages langagiers, mais à la manière de penser et d'utiliser la compréhension orale dans toutes les disciplines. Elle les rend autonomes dans leurs apprentissages et dans l'intégration de leurs nouvelles connaissances, sachant que plus 
de $75 \%$ des enseignements se réalisent en contexte de compréhension orale.

Les résultats de cette recherche montrent aussi que dans la majorité des situations scolaires vécues par les élèves de cette recherche, ces derniers mobilisent une stratégie plus qu'une autre, soit la prise de notes, mais pas dans un objectif de structuration des informations entendues. En effet, cette stratégie sert essentiellement à se remémorer les informations entendues et à les redire textuellement, sans leur accorder de traitement ou même sans considérer le contexte d'énonciation. Cette stratégie serait aussi la plus encouragée par les enseignants. Pourtant, dans les sections des documents ministériels qui se rapportent à l'écriture, il est précisé que, pour informer, il importe de reformuler, d'utiliser des synonymes, etc. afin de montrer une appropriation des informations. Or, les informations manquent quant à l'enseignement et à la pertinence d'une stratégie de prise de notes pour structurer et organiser la compréhension orale, mais surtout en ce qui concerne les liens entre la compréhension de l'oral et celle de l'écrit pour diminuer la surcharge cognitive vécue par certains élèves en raison des caractéristiques de l'oral qui ne repose pas seulement sur les mots. L'enseignement d'une stratégie de prise de notes permettrait de faire contraster l'écrit avec l'oral pour les élèves en posant des questions telles «La forme orale est plus longue que la présentation écrite: à quoi est-ce dû?», "Qu'est-ce qui remplace, à l'oral, la mise en page de l'écrit: changement de paragraphe, caractère gras...?» (Parpette, 2008, pp. 6-7), mais permettrait aussi de cerner que les caractéristiques du genre, informatif, dans le cas qui nous occupe, ne sont pas si éloignées à l'oral et à l'écrit. Ceci étant, leur montrer clairement les distinctions entre les deux présenterait aussi les caractéristiques spécifiques de l'oral et de l'écrit (Nonnon, 2004) pour mieux comprendre ce couple si soudé et ainsi réaliser un véritable travail sur le triptyque qui compose la compréhension orale d'un document audiovisuel à savoir le verbal, le non verbal et le prosodique. 


\section{Notes}

1 Un exemple de grille d'écoute remplie par Constance est fourni en annexe.

2 http://www1.education.gouv.qc.ca/progressionPrimaire/francaisEns/index. asp?page=util_lire

3 Les caractéristiques du genre informatif sont enseignées en écriture aux élèves dès le premier cycle du primaire avec les textes qui "décrivent» (MELS, 2009). Il en va de même pour la lecture et la compréhension en lecture de textes informatifs. Ces textes et leurs caractéristiques sont réinvestis tout au long du primaire. Or, on ne retrouve pas leurs caractéristiques dans les prescriptions ministérielles en ce qui concerne les documents oraux. Pourtant, on présente des documentaires aux élèves, des chroniques variées et on leur demande de produire oralement des oraux qui s'y rattachent ou qui abordent la description d'un pays, d'un métier, etc. Une entrée par les genres aiderait à mieux comprendre les caractéristiques qui sont propres et communes au volet oral et au volet écrit.

4 L'intention projet d'écoute du premier projet était: «Informer la chercheuse du sujet de la vidéo en s'appuyant seulement sur les informations du document audiovisuel».

5 Pour les besoins de cette contribution, seules les données liées au premier projet d'écoute réalisé par les élèves sont abordées. Ces dernières sont choisies, car elles présentent un portrait fidèle des verbalisations à haute voix qu'ont aussi tenu les élèves pour les autres projets d'écoute. En effet, les élèves ont surtout verbalisé des stratégies associées à la prise de notes pour retenir les informations.

6 La verbalisation à haute voix sans rappel est celle qui s'apparente le plus à une situation ordinaire d'écoute en salle de classe. Les élèves ont regardé, de manière individuelle, une vidéo informative traitant d'un sujet qu'ils connaissent et qui est d'intérêt pour eux, selon leurs dires. Cette technique de verbalisation présuppose la possibilité de rappeler à la mémoire de travail des informations traitées et stockées dans la mémoire à long terme de manière autonome (Vermersch, 2011). Ce type de verbalisation à haute voix a permis de solliciter les commentaires des élèves à propos du déroulement de la tâche après sa réalisation.

7 Voici les sujets des vidéos pour chacun des élèves. Charlotte: le violon; Marguerite: les arts de la scène; Simone: l'escalade; Constance: les arts de la scène; Octave: le soccer féminin; Claire: les arts de la scène. La durée des vidéos visionnées variait entre six minutes quinze secondes (00:06:15) et sept minutes vingt secondes (00:07:20). Les documents ont été choisis et validés, car ils présentaient un lexique adapté aux élèves, leur visuel était similaire (un protagoniste qui monologue sur le sujet et qui l'illustre à l'aide de quelques plans visuels simples) et les éléments prosodiques appropriés (le débit était ni trop long ni trop lent, l'intonation variée, etc.).

8 http://www1.education.gouv.qc.ca/progressionPrimaire/francaisEns/index. asp?page $=$ compApp

9 Trouble déficitaire de l'attention.

10 http://www1.education.gouv.qc.ca/progressionPrimaire/science/index. asp?page $=$ strategies 3

$11 \mathrm{http} / / / \mathrm{www} 1$. ducation.gouv.qc.ca/progressionPrimaire/mathematique/index. asp?page $=\mathrm{ex}-$ Strategies

12 http://www1.education.gouv.qc.ca/progressionPrimaire/francaisEns/index. asp? page $=$ conn - _lire

13 http://www1.education.gouv.qc.ca/progressionPrimaire/mathematique/index. asp?page $=$ ex - Strategies 


\section{Annexe:}

Grille d'écoute remplie portant sur l'escalade

\begin{tabular}{|c|c|}
\hline \multirow{2}{*}{\multicolumn{2}{|c|}{$\begin{array}{c}\text { Grille d'écoute pour le premier projet d'écoute portant sur un sujet connu des élèves } \\
\text { Intention d'écoute } \\
\text { Informer la chercheure au sujet de leur vidéo en s'appuyant seulement sur les } \\
\text { informations du document audiovisuel }\end{array}$}} \\
\hline & \\
\hline \multicolumn{2}{|c|}{ Préécoute (avant le visionnement) } \\
\hline \multicolumn{2}{|c|}{$\begin{array}{l}\text { Qu'est-ce que je connais déjà du sujet X? Qu'elles informations j'aimerais avoir sur le } \\
\text { sujet X? }\end{array}$} \\
\hline $\begin{array}{l}\text { Ce que je connais déjà sur le sujet: } \\
\text { Les lyypes d'senaluches } \\
\text { t nom des puses } \\
\text { L es nom des partio du } \\
\text { harnais }\end{array}$ & $\begin{array}{l}\text { Mes hypothèses sur ce qui sera dit dans le } \\
\text { document X: } \\
\text { des bases } \\
\text { - commint essurin }\end{array}$ \\
\hline \multicolumn{2}{|c|}{ Écoute } \\
\hline Thème : sujet $\mathrm{X}$ & \\
\hline \multicolumn{2}{|l|}{ Une stratégie quime sera utile : for } \\
\hline Élêment & ions \\
\hline 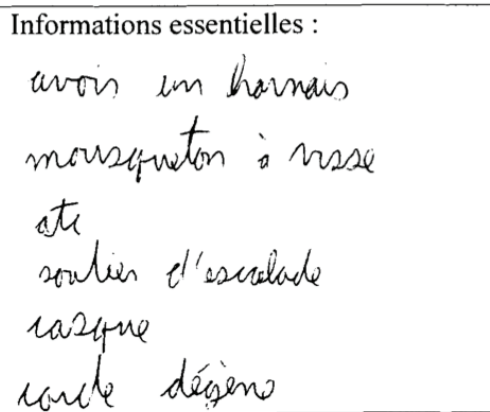 & 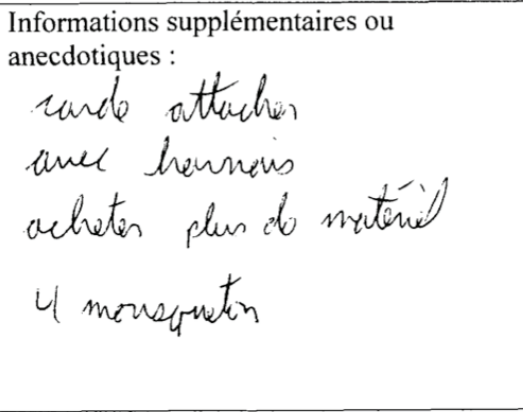 \\
\hline $\begin{array}{l}\text { Élément (s) à approfondir: } \\
\text { esculacte en tato } \\
\text { bout do curdeletto }\end{array}$ & Une question que je me pose : \\
\hline
\end{tabular}




\section{Bibliographie}

Allen, N. (2017a). Verbalisation de stratégies de compréhension orale dans des projets d'écoute en français langue d'enseignement, par des élèves du $3^{e}$ cycle du primaire. Thèse de doctorat inédite, Université du Québec à Montréal en collaboration avec l'Université du Québec en Outaouais.

Allen, N. (2017b). La prise de notes: une stratégie répandue en situations pédagogiques de compréhension orale, Repères, 54, 39-59. DOI: 10.4000/reperes.1081

Beacco, J.-C. \& Porquier, R. (2007). Niveau A1 pour le français. Un référentiel. Paris: Didier.

Berner, C. \& Thouard, D. (2008). Sens et interprétation: pour une introduction à l'herméneutique (Coll. «Opuscules, 23»). Villeneuve-d’Ascq, France: Presses universitaires du Septentrion.

Bétrix Köhler, D., Nidegger, C., Revaz, N., Riesen, W. \& Wirthner, M. (1999). Et si les pratiques enseignantes en production écrite nous étaient contées...: des discours aux activités, en classe de Ge. Neuchâtel: IRDP; Le Mont-sur-Lausanne: Loisirs et pédagogie (LEP).

Bouffard, T. (1998). A developmental study of the relationship between reading development and the self-system. European Journal of Psychology of Education, 13, 61-74.

Bronckart, J.-P. (1996): Activité langagière, textes et discours. Pour un interactionisme socio-discursif. Neuchâtel-Paris: Delachaux et Niestlé.

Brown, A. L. \& Campione, J. C. (1995). Concevoir une communauté de jeunes élèves. Leçons théoriques et pratiques. Revue française de Pédagogie, 11, 11-33.

Cain, K., Oakhill, J. V., Barnes, M. A. \& Bryant, P. E. (2001). Comprehension skill, inference-making ability, and their relation to knowledge. Memory \& Cognition, 29, (6), 850-859. Consulté dans http://www.springerlink.com/index/P1737282P1J7Q107.pdf

Chartrand, S.-G., Émery-Bruneau, J. \& Sénéchal, K. (avec Riverin, P.). (2015). Caractéristiques de 50 genres pour développer les compétences langagières en français au secondaire. Québec, QC: Didactica.

Chinn, C. \& Chinn, L. (2009). Cognitive strategies. In E. M. Anderman \& L. H. Anderman (Éd.), Psychology of classroom learning: an encyclopedia (pp. 467-563). Detroit, MI: Macmillan Reference USA, Gale Cengage Learning. Format ePub.

Chiss, J.-L. (2002). Le couple orallécrit et la tension entre communicatif et cognitif. Présentation lors du colloque international de Didactique de l'oral, Ministère de l'Éducation nationale, Paris. Consulté dans http://eduscol.education.fr/cid46393/le-couple-oral-ecrit-et-la-tension-entre-communicatif-et-cognitif.html

Conseil supérieur de la langue française. (2015). Rehausser la maitrise du français pour raffermir la cohésion sociale et favoriser la réussite scolaire. Québec, QC: Collectif. Récupéré de http:// www.cslf.gouv.qc.ca/publications/avis207/a207.pdf

Cornaire, C. \& Germain, C. (1998). La compréhension orale. Baume-les-Dames, France: CLE International.

De Pietro, J.-F. \& Whirthner, M. (1996). Oral et écrit dans les représentations des enseignants et dans les pratiques quotidiennes de la classe de français. Tranel, 25, 29-49.

Dolz, J. \& Schneuwly, B. (1998). Pour un enseignement de l'oral; Initiation aux genres formels à l'école. Paris: ESF éditeur.

Efklides, A. (2008). Metacognition: Defining its facets and levels of functioning in relation to self-regulation and co-regulation. European Psychologist, 13, 277-287.

Fayol, M. \& Gombert, J.-M. (1992). Psychologie cognitive de la lecture (Coll. «Psychologie d'aujourd'hui»). Paris: Presses universitaires de France.

Field, J. (2013). Cognitive validity. Dans A. Geranpayeh \& L. Taylor (Éd.), Examining Listening (pp. 77-15). Cambridge, England: Cambridge University Press.

Flavell, J. H. (1985). Développement métacognitif. Dans J. Bideaud \& M. Richelle (Éd.), Psychologie développementale. Problèmes et réalités (pp. 29-41). Bruxelles: Mardaga.

Garcia-Debanc, C. \& Plane, S. (2004). Comment enseigner l'oral à l'école primaire? Paris: Hatier. 
Graham, C. R. (2005). Blended learning systems: Definition, current trends, and future directions. Dans C. J. Bonk \& C. R. Graham (Éd.), Handbook of blended learning: Global perspectives, local designs (pp. 3-21). San Francisco, CA: Pfeiffer.

Graham, S., Santos, D. \& Vanderplank, R. (2011). Exploring the relationship between listening development and strategy use. Language Teaching Research, 15, (4), 435-456. Consulté dans http://ltr.sagepub.com/content/15/4/435.short

Guthrie, J. T., Wigfield, A., Metsala, J. L. \& Cox, K. E. (1999). Motivational and cognitive predictors of text comprehension and reading amount. Scientific Studies of Reading, 3, 231-256.

Hartman, H. J. (2001). Metacognition in learning and instruction: Theory, research and practice. New York, NY: Springer.

Lafontaine, L. (2007). Enseigner l'oral au secondaire. Séquences didactiques intégrées et outils d'évaluation. Montréal, QC: Chenelière Éducation.

Lazure, R. (1992). Vers une didactique du français oral: État de question des recherches menées entre 1970 et 1990. Thèse de doctorat inédite, Université de Montréal.

Legendre, R. (2005). Dictionnaire actuel de l'éducation (3e éd.). Montréal: Guérin.

Letarte, A. \& Lafond, F. (2001). L'écoute en classe et la prise de notes (4e éd.). Québec, QC: Université Laval. Consulté dans https:/www.aide.ulaval.ca/files/content/sites/aide/files/ files/reussite/Guide_Ecoute.pdf

Lhote, E. (1995). Enseigner l'oral en interaction. Percevoir, écouter, comprendre. Paris: Hachette.

Ministère de l'Éducation du Loisir et du Sport. (MELS). (2006). Programme de formation de l'école québécoise. Enseignement primaire. Québec, QC: Gouvernement du Québec.

Ministère de l'Éducation du Loisir et du Sport. (MELS). (2009). Progression des apprentissages au primaire. Québec, QC: Gouvernement du Québec.

Ministère de l'Éducation du Loisir et du Sport. (MELS). (2011). Progression des apprentissages en communication orale au primaire. Québec, QC: Gouvernement du Québec.

Nolin, R. (2013). Pratiques déclarées d'enseignement et d'évaluation de l'oral d'enseignants $d u$ primaire au Québec. Mémoire de maitrise inédit, Université du Québec à Montréal.

Nonnon, É. (2002). Des interactions entre oral et écrit: notes, canevas, traces écrites et leurs usages dans la pratique orale. Pratiques, 115-116. Consulté dans http://pratiques-cresef. fr/p115_no1.pdf

Nonnon, É. (2004). Écouter peut-il être un objectif d'apprentissage? Le Français aujourd'hui, $146,75-84$.

OCDE. (2014). Regards sur l'éducation 2014: Les indicateurs de l'OCDE. Éditions OCDE. Consulté dans http://www.oecd.org/edu/Regards-sur-l'education-2014.pdf

Paillé, P. \& Muchielli, A. (2012). L'analyse qualitative en sciences humaines et sociales. Paris: Armand Colin.

Parpette, C. (2008). De la compréhension orale en classe à la réception orale en situation naturelle: une relation à interroger. Recherches en Didactique des Langues et Cultures: les Cahiers de l'acedle, 5, (1), 219-232.

Réseau canadien de recherche sur le langage et l'alphabétisation. (RCRLA). (2009). Stratégie nationale d'alphabétisation précoce: Rapport et recommandations. Récupéré de http://docs. cllrnet.ca/NSEL/finalReportFR.pdf

Romainville, M. (2000). La métamémoire. Dans L. Lafortune, P. Mongeau \& R. Pallascio (Éd.), Métacognition et compétences réflexives (pp. 223-244). Montréal, QC: Logiques.

Rost, M. (2002). Teaching and Researching Listening. Grande-Bretagne: Pearson Education.

Sénéchal, K. \& Chartrand, S.-G. (2011). État des lieux de l'enseignement du français (ELEF). Les Cahiers de l'AQPF, 1, (1), 4-5. Consulté dans http://tesl-ej.org/pdf/ej64/r4.pdf

Vandenrdorpe, C. (1992). Comprendre et interpréter. Dans C. Préfontaine \& M. Lebrun (Éd.), La lecture et l'écriture enseignement et apprentissage: actes du colloque. Montréal, QC: Éditions Logiques. 
Van der Maren, J.-M. (2010). Les recherches qualitatives: des critères variés de qualité en fonction des types de recherche. Dans L. Paquay, M. Crahay \& J. M. De Ketele (Éd.), L'analyse qualitative en éducation: des pratiques de recherche aux critères de qualité (pp. 69-84). Bruxelles: de Boeck.

Vandergrift, L. \& Goh, C. M. (2012). Teaching and Learning Second Language Listening. Metacognition in Action. New York, NY: Routledge.

Vermersch, P. (2011). L'entretien d'explicitation. Issy-Les-Moulineaux, France: ESF.

Wallace, J. \& Louden, W. (2003). What we don't understand about teaching for understanding: Questions from science education. Journal of Curriculum Studies, 35, (5), $545-566$

Mots-clés: Compréhension orale, prise de notes, enseignement du genre, stratégies, verbalisation à haute voix

\section{Mündliches und schriftliches Verstehen: Wie können Verbindungen verstärkt und Eigenheiten bewahrt werden?}

\section{Zusammenfassung}

Der Zusammenhang von mündlichem und schriftlichem Verstehen ist unantastbar, obschon diese unterschiedliche Charakteristiken aufweisen. In diesem Artikel wird beabsichtigt, die im Rahmen eines Hörprojekts von sechs Schülerinnen und Schülern der sechsten Klasse (11-12 Jahre) einer Grundschule in Quebec verbalisierten metakognitiven Strategien qualitativ zu beschreiben. In diesem Projekt wurden die Schülerinnen und Schüler dazu angeleitet, ein Raster zur Dokumentation ihres Hörverständnisses zu benutzen. Die Verbalisierungen zeigen, dass sie vor allem eine Notiz-Strategie zum Behalten von Informationen verwenden. Die Daten zeigen die Möglichkeit auf, diese Strategie in einer transdisziplinären Perspektive von Bildung zu verwenden.

Schlagworte: Hörverstehen, Notizen anfertigen, Unterricht zu Textgenre, Strategien, lautes Denken 


\section{Comprensione orale e comprensione scritta: come rafforzare i loro legami conservandone le specificità?}

\section{Riassunto}

Il rapporto tra comprensione orale e scritta è innegabile, benché ad esse si possano assegnare caratteristiche distinte. In questo articolo intendiamo descrivere in modo qualitativo le strategie metacognitive verbalizzate da 6 allievi frequentanti il sesto anno della scuola primaria in Quebec (11-12 anni), coinvolti in un progetto di comprensione orale. In tale contesto essi sono stati guidati ad utilizzare una scheda per rendere conto della loro comprensione orale. Le loro verbalizzazioni ci informano di come essi utilizzino essenzialmente una strategia di annotazione per trattenere le informazioni. Questi dati ci aiutano a riflettere sull'uso di tale strategia in una prospettiva di insegnamento transdisciplinare.

Parole chiave: comprensione orale, annotazione, insegnamento delle tipologie di testo, strategie, verbalizzazione ad alta voce

\section{Listening Comprehension and Written Comprehension: How to Solidify Their Links While Maintaining Their Specificities?}

\section{Abstract}

The links between listening and reading comprehension are undeniable, although distinct characteristics are imputed to them. In this article, we intend to describe qualitatively the metacognitive strategies verbalized by six pupils of sixth grade (11-12 years) of Quebec elementary school in a listening project. In the latter, they used a grid to account for their listening comprehension. Their verbalizations tell us that they essentially use a note-taking strategy to retain information. This data helps us to think about the use of this strategy in a transdisciplinary perspective of education.

Keywords: Listening comprehension, note-taking, text type teaching, strategies, think aloud 